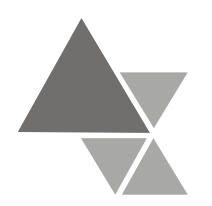

SEGURANCA

\title{
Segurança Alimentar em uma Comunidade Rural no Alto Vale do Jequitinhonha/Mg
}

\author{
Luciana Neri Nobre ${ }^{1}$, Nadja Maria Gomes Murta ${ }^{2}$, Marcelo Mandacaru Souza ${ }^{3}$, Nayara Cristiane \\ Ferreira $^{3}$, Leandro de Morais Cardoso ${ }^{3}$, Fabiana Rossi Hamacek ${ }^{3}$
}

\section{Resumo}

O presente estudo buscou descrever a situação de segurança alimentar vivenciada por famílias residentes na comunidade rural de Galheiros do município de Diamantina, situada no Alto Vale do Jequitinhonha, no Estado de Minas Gerais, Brasil. Foi utilizada a Escala Brasileira de Insegurança Alimentar - EBIA - com 15 questões que refletem a insegurança alimentar em diferentes níveis de intensidade. Foram investigadas 28 famílias, e obtidas informações sobre renda familiar, renda per capita, condição sócio econômica, de moradia, de saneamento básico e consumo alimentar. A prevalência de insegurança alimentar encontrada na comunidade foi de 46,4\%, sendo 32,14\% leve, $3,6 \%$ moderada e $10,7 \%$ grave. Mais da metade das famílias $(57,2 \%)$ viviam com o equivalente a $1 \frac{1}{2}$ do salário mínimo por mês. A maioria das famílias $(75,0 \%)$ relatou gastar com alimentação valores inferiores a R \$350,00, e $53,6 \%$ relataram receber da união e/ou Estado algum tipo de benefício. Estes resultados demonstram que apesar de existir no Brasil políticas públicas de combate à fome, grande parte das famílias residentes em Galheiros, vive em vulnerabilidade social, assim sendo, são necessárias ações estruturantes para reverter tal quadro, tanto no que se refere à produção de alimentos, quanto ao acesso a alimentos em qualidade e quantidade suficiente.

Palavras chave: segurança alimentar, comunidade rural, vulnerabilidade social, renda familiar

\begin{abstract}
This present study objectived to describe the food security condition, economic conditions, infrastructure and food intake of families who live in a rural community - Galheiros- localized in Alto Vale do Jequitinhonha, of the Minas Gerais state, Brazil. It was used the Escala Brasileira de Insegurança Alimentar - EBIA -, adapted, with 15 questions that reflect the food insecurity in different levels of intensity. Twenty eight families were investigated, and were obtained information about them, such as familiar income, per capita income, socio-economic conditions and housing, basic sanitation and their food intake. The prevalence of families with some level of food insecurity was $46.4 \%$, witch $32.1 \%$ of them were on low insecurity level, $3.6 \%$ moderate insecurity level and $10.7 \%$ on serious insecurity level. More than the half of the families $(57.1 \%$ ) declared to live with $1 / 2$ of the minimum salary or less than that. The majority $(75.0 \%)$ declared expend with food on average $\mathrm{R} \$ 350.00 ; 53.6 \%$ to receive from government some type of benefit. These results show that although to exist in Brazil public politics to combat to the hunger, most of the families living in Galheiros, shows in social vulnerability, therefore, public actions are needed to reverse this situation, both as regards the of food production as access to food in sufficient quantity and quality.
\end{abstract}

Keywords: food security, rural community, social vulnerability, familiar income

\footnotetext{
${ }^{1}$ Docente do curso de Nutrição da Universidade Federal dos Vales do Jequitinhonha e Mucuri - UFVJM, doutoranda do Programa de pós-graduação em Ciências da Saúde pela Universidade Federal de Minas Gerais. Rua da Glória, 187, Centro, sala 34 - Diamantina - MG - CEP: 39100-000, fax: (38) 3531-1030 - E--mail: lunerinobre@ yahoo.com.br.

${ }^{2}$ Docente do curso de Nutrição da Universidade Federal dos Vales do Jequitinhonha e Mucuri - UFVJM, doutoranda do Programa de Estudos Pós-graduados em Ciências Sociais/Antropologia pela Pontificia Universidade Católica de São Paulo.

${ }^{3}$ Nutricionistas pela Universidade Federal dos Vales do Jequitinhonha e Mucuri - UFVJM.
} 


\section{Introdução}

Atualmente, várias são as discussões em torno do tema segurança alimentar, pois a mesma inclui diferentes aspectos, tais como: produção e iniqüidades na distribuição de alimentos, consumo, valorização e respeito aos hábitos alimentares das populações tradicionais, dentre outros. Dada esta abrangência, a partir da realização da Segunda Conferência Nacional de Segurança Alimentar e Nutricional, em 2004, o seguinte conceito foi adotado no país:

a realização do direito de todos ao acesso regular e permanente a alimentos de qualidade, em quantidade suficiente, sem comprometer o acesso a outras necessidades essenciais, tendo como base práticas alimentares promotoras de saúde, que respeitem a diversidade cultural e que sejam social, econômica e ambientalmente sustentáveis[1].

Para Valente ${ }^{[2]}$ o direito à alimentação adequada é um direito humano básico reconhecido no Pacto Internacional de Direitos Econômicos, Sociais e Culturais. Sem ele, não se podem discutir os demais:

Sem uma alimentação adequada, tanto do ponto de vista de quantidade como de qualidade, não há o direito à vida. Sem uma alimentação adequada, não há o direito à humanidade, entendida aqui como direito de acesso à vida e à riqueza material, cultural, científica e espiritual produzida pela espécie humana.

Entre as ações para garantia da SA, de acordo com o CONSEA $^{[3]}$, pode-se citar o incentivo à agricultura familiar. As famílias rurais que fazem uso deste tipo de agricultura consideram-na um importante componente de sua alimentação, reprodução econômica e principal referência de identidade social. Identidade esta, heterogênea no país em decorrência das diferentes trajetórias históricas dos grupos sociais, bem como da diversidade dos ecossistemas, dos modelos produtivos e do grau de integração com a agroindústria ${ }^{[4]}$.

A insegurança alimentar (IA) ocorre quando um indivíduo, ou grupo populacional, não tem acesso de forma regular e permanente aos alimentos em termos qualitativos e quantitativos $^{[5]}$. Entre as diversas causas destacam-se as desigualdades sociais, a concentração de renda e dos meios de produção. Embora a insegurança alimentar não se manifeste exclusivamente nas camadas sociais mais pobres, é nestes grupos que encontramos o maior grau de vulnerabilidade à falta de alimentos, seja por não disporem de renda suficiente para aquisição dos mesmos, seja por não terem acesso aos bens de produção necessários: terra, insumos, equipamentos, etc.

Os instrumentos de medidas de insegurança alimentar, ao serem adotados em um país, devem ser capazes de refletir os diferentes níveis e possibilidades de acesso aos alimentos, vivenciados pelas famílias. Para tal, o instrumento de medida deve ser simples, de fácil aplicação, de compreensão o mais universal possível, de baixo custo e de fácil análise $\mathrm{e}^{[6,7,8]}$.

Em concordância com esta perspectiva a Escala Brasileira de Segurança Alimentar (EBIA) foi validada ${ }^{[5,7,8,9]}$, sendo este instrumento também recomendado para a avaliação do impacto de políticas públicas 
sobre a condição de acesso da população à alimentação adequada.

Classificam-se as famílias como com segurança alimentar (SA) quando não há problemas de acesso aos alimentos em termos qualitativos ou quantitativos e não há preocupação de que os alimentos venham a faltar no futuro. A insegurança alimentar leve (IAL) ocorre quando há preocupação com a falta de alimentos no futuro próximo (componente psicológico da insegurança) e há arranjos domésticos para que os alimentos durem mais. A moderada (IAM) ocorre quando há situação de comprometimento da qualidade da alimentação na busca de manter a quantidade necessária. Neste nível de insegurança, inicia-se a redução da quantidade de alimentos entre os adultos da família. E, finalmente, a grave (IAG) é a condição em que há deficiência quantitativa de alimento levando à situação de fome entre adultos e crianças da família ${ }^{[5,7,8,9,10]}$.

No país, em 2004, de acordo com a Pesquisa Nacional por Amostras de Domicílio - PNAD $^{[5]}, 65,2 \%$ das famílias pesquisadas estavam em situação de segurança alimentar, $16 \%$ conviviam com a insegurança alimentar leve, $12,3 \%$ com a moderada e $6,5 \%$ com a grave. Para o Brasil rural os valores encontrados foram respectivamente: $56,5 \%$, $17,4 \%, 17 \%$ e $9,0 \%$ (menor prevalência de SA e maior prevalência de IA).

Neste contexto, estudos foram realizados no Brasil para avaliação do nível de segurança alimentar de sua população ${ }^{[5,8,9,10,11,12,]}$. Essas pesquisas têm mostrado resultados bastante preocupantes grande parte da população brasileira está vivendo em insegurança alimentar. Yuyama et $a l^{[9]}$, ao avaliarem a segurança/insegurança alimentar de famílias urbanas e rurais no estado do Amazonas, encontraram que apenas $10,9 \%$ e $31,3 \%$ da população urbana e rural respectivamente viviam com segurança alimentar. Pereira et $a l^{[11]}$ observaram que, entre famílias de alta vulnerabilidade social da cidade de São Paulo, a situação de segurança alimentar foi observada em apenas $12 \%$ das famílias estudadas.

Em recente publicação do Instituto Brasileiro de Análises Sociais e Econômicas $\left(\right.$ IBASE) ${ }^{[12]}$ sobre a repercussão do programa bolsa família na segurança alimentar das famílias beneficiadas, foi constatado que, entre as mesmas, $83 \%$ viviam em situação de insegurança alimentar, sendo $28 \%$ leve, $34 \%$ moderada e $21 \%$ grave.

Tendo em vista os aspectos acima discutidos, a presente pesquisa objetivou traçar o perfil de segurança alimentar, de condições econômicas, de infra-estrutura e de consumo alimentar das famílias residentes na comunidade de Galheiros, Minas Gerais.

\section{Casuística e Métodos}

Foi realizado um estudo transversal descritivo no qual a coleta dos dados ocorreu durante visitas realizadas, aos finais de semana, às residências de cada família durante os meses de outubro e novembro de 2006.

Foram eleitas para participarem dessa pesquisa todas as famílias residentes em Galheiros, uma comunidade rural do município de Diamantina localizada no Alto Vale do Jequitinhonha-MG, composta por 30 famílias. Essa comunidade apresenta 
características peculiares, como trabalho de coleta de flores "sempre vivas" (refere-se ao nome genérico das espécies da família Eriocaulaceae dado às inflorescências e escapos que mesmo depois de colhidas conservam a forma e a cor por um longo período de tempo) para confecção de artesanatos. As culturas possuem característica tipicamente agro-familiar, sendo que o extrativismo da "sempre viva", atualmente, apresenta um caráter predominantemente social por absorver mão-de-obra em todas as operações, desde a colheita até a exportação, destacando como forma de geração de renda para as famílias em que se emprega mão-deobra familiar. Destaca-se também a prática da agricultura familiar sendo que esta é, em sua predominância, de subsistência.

Para medir o nível de segurança alimentar das famílias residentes em Galheiros, utilizou-se a EBIA ${ }^{[5,8,9,10]}$, trata-se de um questionário com 15 perguntas fechadas, com respostas afirmativas (sim) e negativas (não). Na totalidade das perguntas sete são direcionadas apenas às famílias onde há moradores menores de 18 anos. Cada resposta afirmativa corresponde a 1 ponto, a soma obtida representa a pontuação da escala, cuja amplitude varia de 0 a 15 pontos. Os resultados obtidos são assim categorizados: SA nenhuma resposta positiva; IAL: 1-5 pontos em famílias com menores de 18 anos ou 1-3 pontos nas sem menores; IAM: 6-10 ou 4-6 pontos; e IAG: 11-15 ou 7-8 pontos $[5,8,9,10,11]$

Para avaliar as condições econômicas, de infra-estrutura e de consumo alimentar, utilizou-se da metodologia proposta por Galleazi $^{13}$, cujo instrumento é composto pelas seguintes variáveis: composição familiar (sexo, idade, grau de escolaridade e estado fisiológico), gasto mensal com refeições, recebimento de doação de algum tipo de alimento no último mês, tipo de doação recebida, origem da doação (prefeitura, governo federal, dentre outras), dados de infraestrutura (profissão do chefe da família, renda familiar, tipo de construção e situação da moradia, saneamento e bens da família) e consumo alimentar avaliado por um questionário de freqüência semanal.

Foram realizados os seguintes procedimentos: a renda de cada membro da família foi questionada em Reais; a variável renda familiar mensal correspondeu à soma das rendas individuais sendo transformada em faixas de salário mínimo para algumas análises (salário mínimo vigente naquele ano era de $\mathrm{R} \$$ $350,00)$. O consumo semanal de alimentos foi indagado de forma qualitativa, questionando a freqüência de consumo (raramente, uma a quatro vezes por semana e cinco ou mais vezes por semana) pelas famílias para os grupos das carnes, leite e derivados, ovos, legumes, verduras, arroz e feijão, óleo/margarina, frutas, suco de frutas. Não foi possível realizar o questionário, individualmente, como normalmente é feito, ele foi aplicado apenas aos responsáveis pelo domicílio, sendo questionados quais grupos de alimentos eram consumidos, semanalmente, em suas casas, e se todos da família os consumiam.

Essa pesquisa foi realizada após aprovação do Comitê de Ética na Pesquisa da Universidade Federal dos Vales do Jequitinhonha e Mucuri, e somente participaram do estudo as famílias que assinaram o Termo de Consentimento Livre e 
Esclarecido (TCLE) aceitando participar do estudo.

O teste de Fisher (Fisher's exact test), com nível de significância de 5\%, foi utilizado para avaliar a associação entre a situação de segurança alimentar e as variáveis: renda, presença de crianças/adolescentes na família, número de moradores por domicílio, recebimento de algum benefício do Governo Estadual e Federal, além da frequiência de consumo alimentar de alguns grupos de alimentos. As análises foram realizadas com o software $S A S^{[14]}$

\section{Resultados e Discussão}

Dentre as famílias eleitas para o estudo, foram pesquisadas 28 , totalizando 100 habitantes, o que corresponde a $93,3 \%$ das famílias da comunidade de Galheiros. Duas não foram contatadas por dificuldades de acesso dos pesquisadores as suas residências em decorrência de chuvas intermitentes no período da coleta dos dados.

Para a avaliação do nível de segurança alimentar (Tabela 1), os valores encontrados foram comparados com os resultados da PNAD $/ 2004^{[5]}$ referentes ao Brasil, nordeste e sudeste rural. Justifica-se tal comparação pelo fato de supormos que a região do Vale do Jequitinhonha tenha características socioeconômicas semelhantes às do nordeste brasileiro, embora, em termos geográficopolítico, esteja localizada na região sudeste.

Tabela 1. Distribuição de domicílios particulares em situação de segurança/insegurança alimentar, segundo Regiões da Federação e da comunidade de Galheiros, Minas Gerais, 2006.

\begin{tabular}{l|c|c|c|c|c}
\hline \multirow{2}{*}{$\begin{array}{c}\text { Unidades da } \\
\text { Federação }\end{array}$} & \multicolumn{5}{c}{ Domicílios Particulares } \\
\cline { 2 - 6 } & Com SA (\%) & \multicolumn{4}{c}{ Com IA } \\
\cline { 3 - 6 } & & Total (\%) & Leve (\%) & Moderada (\%) & Grave (\%) \\
\hline Brasil Rural* & 56,5 & 43,4 & 17,4 & 17,0 & 9,0 \\
Sudeste Rural* & 64,4 & 35,5 & 20,1 & 11,6 & 3,8 \\
Nordeste Rural* & 35,0 & 65,0 & 20,8 & 27,1 & 17,1 \\
Galheiros & 53,6 & 46,4 & 32,1 & 3,6 & 10,7 \\
\hline
\end{tabular}

Fontes: * PNAD (2004) publicados pelo IBGE (2006).

De acordo com a Tabela 1, 53,6\% das famílias de Galheiros estavam em situação de segurança alimentar semelhante ao encontrado no Brasil rural $(56,5 \%)$, e em situação intermediária entre o nordeste e sudeste rural: $35,0 \%$ e $64,4 \%$ respectivamente. No entanto, encontram-se em melhor situação que as famílias de uma região de alta vulnerabilidade social da cidade de São Paulo na qual foi observada situação de SA em apenas 12,0\%, como apontado por Pereira $e t a l^{[11]}$.

Entre as famílias que conviviam com a IA (Tabela 1), observa-se que $32,1 \%$ preocupavam-se com uma possível falta de alimentos (IAL); 3,6\% estavam em situação de 
IAM, afetando, possivelmente, a quantidade dos alimentos ingeridos e 10,7\% conviviam com a restrição quantitativa de alimentos ou com a fome (IAG). Observa-se ainda, que a prevalência de IAL foi superior a todas encontradas pela PNAD/2004 $4^{[5]}$ e que o valor encontrado para IAG foi superior ao sudeste e Brasil rural, 3,8\% e 9,0\%, respectivamente.

Em pesquisa realizada com famílias rurais, na Paraíba ${ }^{[15]}$ observou-se prevalência de SA em 44,5\% das famílias, bem como $14,0 \%$ de IAG. Tal descoberta ajuda a reforçar a hipótese de que as comunidades rurais pertencentes ao Vale do Jequitinhonha assemelham-se mais às características rurais do nordeste brasileiro, do que às do sudeste. Entretanto, as prevalências de IAG encontradas para as famílias rurais da Paraíba $(14,0 \%)$ e de Galheiros (10,7\%), quando comparadas com as famílias urbanas paulistas de alta vulnerabilidade $(15,7 \%)^{[11]}$, estão em melhor situação.

De acordo com Sen ${ }^{[16]}$ mesmo sem "passar fome", muitas famílias podem vivenciar situações de insegurança alimentar, pois a alimentação pode não ser adequada do ponto de vista sanitário, nutricional ou cultural (como os programas que distribuem alimentos não condizentes à cultura alimentar), ou porque os gastos com alimentos comprometem o atendimento de outras necessidades essenciais. Por outro lado, mesmo segmentos situados num patamar de renda que lhes permita uma condição melhor de acesso aos alimentos podem estar submetidos a relações de produção e fatores conjunturais que levem a um quadro de vulnerabilidade alimentar, devido, por exemplo, às oscilações de mercado.

Os resultados da análise da relação entre o número de moradores por domicílio e a situação de segurança alimentar entre as famílias sem e com, pelo menos, um morador menor de 18 anos estão apresentados na Tabela 2. Observa-se que entre as famílias estudadas, $78,6 \%$ tinham moradores com idade inferior a dezoito anos e destas, metade estava em situação de SA. Entre os domicílios com apenas moradores maiores de 18 anos, $60,0 \%$ estavam com SA e tinham até 3 moradores no domicílio.

Segundo Hoffmann ${ }^{[17]}$ a situação de insegurança alimentar é afetada pela presença, ou não, de pessoas com menos de 18 anos e pelo número de pessoas por domicílio. Assim, quanto maior o número de pessoas menores de 18 anos, ou quanto maior o número de pessoas do domicílio, maior a proporção de insegurança alimentar. Entretanto, no presente estudo não se encontrou associação entre a situação de SA ou IA, o número de moradores e a presença de menores de 18 anos por domicílio ( $p=0,65 ; p=0,31$ e $p=1,00)$, diferindo do encontrado pela PNAD/2004 $4^{[5]}$, o que pode ser explicado, possivelmente, pelo pequeno número de famílias avaliadas neste estudo. 
Tabela 2. Distribuição dos domicílios particulares com e sem, pelos menos, um morador menor de 18 anos de idade, segundo a situação de segurança alimentar e o número de moradores nos domicílios da comunidade de Galheiros, Minas Gerais, 2006.

\begin{tabular}{|c|c|c|c|c|c|}
\hline \multirow{3}{*}{$\begin{array}{c}\text { Presença ou ausência de menores de } 18 \text { anos de acordo com o } \\
\text { número de moradores por domicílios }\end{array}$} & \multicolumn{5}{|c|}{ Domicílios Particulares } \\
\hline & \multicolumn{2}{|c|}{ SA } & \multicolumn{2}{|c|}{ IA } & \multirow[b]{2}{*}{$p$ valor } \\
\hline & $\mathrm{n}$ & $\%$ & $\mathrm{n}$ & $\%$ & \\
\hline Presença de moradores menores de 18 anos & 11 & 50,0 & 11 & 50,0 & $0,65^{1}$ \\
\hline Até 3 moradores & 04 & 80,0 & 01 & 20,0 & $0,31^{2}$ \\
\hline 4 ou mais moradores & 07 & 41,2 & 10 & 58,8 & \\
\hline Ausência de moradores menores de 18 anos & 04 & 66,7 & 02 & 33,3 & \\
\hline Até 3 moradores & 03 & 60,0 & 02 & 40,0 & $1,00^{2}$ \\
\hline 4 ou mais moradores & 01 & 100,0 & 0 & 0 & \\
\hline
\end{tabular}

1. Teste exato de Fisher: presença/ausência de menores de 18 anos e situação de segurança alimentar; 2 . Teste exato de Fisher: número de pessoas na família e situação de segurança alimentar estratificado por presença/ausência de menores de 18 anos.

As características referentes às condições socioeconômicas da comunidade podem ser observadas pela Tabela 3 . Todas as famílias estudadas sempre residiram em Galheiros, sendo que 75,0\% apresentavam um gasto médio mensal com alimentação inferior a um salário mínimo.

No que diz respeito ao recebimento de benefício/auxílio da União/Estado ou de particulares para a alimentação, observou-se que $53,6 \%$ das famílias receberam algum tipo de benefício no último mês antes da coleta dos dados. Tal fato pode ser explicado pela baixa renda associada à presença de crianças no meio familiar. $\mathrm{O}$ benefício recebido com maior frequiência foi o advindo do Programa Bolsa
Família, seguido do Leite pela Vida. O Governo Federal foi o que mais contribuiu para essa ação (Tabela 3). Este resultado se assemelha ao encontrado por Pereira et $a l^{[11]}$ no qual 53,0\% das famílias avaliadas recebiam algum tipo de auxílio ou doação, seja do Governo, de entidade religiosa ou não religiosa.

Ao relacionar a presença de benefício oriundo do Governo Estadual ou Federal, com a situação de SA, também não foram encontradas associações estatisticamente significativas $(p=0,98)$. No entanto, caso a comunidade de Galheiros não fosse beneficiada com estes programas, possivelmente, os valores encontrados de IA poderiam ser superiores. 
Tabela 3. Distribuição das famílias da comunidade de Galheiros, de acordo com algumas variáveis socioeconômicas e situação de Segurança Alimentar. Galheiros, Minas Gerais, 2006.

\begin{tabular}{|c|c|c|c|c|c|c|c|}
\hline \multirow[t]{2}{*}{ Variáveis estudadas } & \multicolumn{2}{|c|}{ Total } & \multicolumn{2}{|c|}{ SA } & \multicolumn{2}{|c|}{ IA } & \multirow[t]{2}{*}{ Valor $p$} \\
\hline & $\mathrm{n}$ & $\%$ & $\mathrm{n}$ & $\%$ & $\mathrm{n}$ & $\%$ & \\
\hline Sempre residiu em Galheiros & & & & & & & $0 * *$ \\
\hline Sim & 28 & 100 & 15 & 53,6 & 13 & 46,4 & \\
\hline Gasto médio mensal com alimentação & & & & & & & 0,39 \\
\hline$<350$ reais & 21 & 75,0 & 10 & 47,6 & 11 & 52,4 & \\
\hline$\geq 350$ reais & 07 & 25,0 & 05 & 71,4 & 02 & 28,6 & \\
\hline $\begin{array}{l}\text { Recebeu alguma ajuda para sua alimentação no } \\
\text { último mês }\end{array}$ & & & & & & & 0,97 \\
\hline Sim & 15 & 53,6 & 08 & 53,3 & 07 & 46,7 & \\
\hline Não & 13 & 46,4 & 07 & 53,8 & 06 & 46,2 & \\
\hline Tipo de benefício recebido & & & & & & & 0,98 \\
\hline Leite pela vida & 05 & 33,3 & 02 & 40,0 & 03 & 60,0 & \\
\hline Leite + bolsa família & 02 & 13,3 & 02 & 100,0 & 0 & 0 & \\
\hline bolsa família & 08 & 53,3 & 05 & 62,5 & 03 & 37,5 & \\
\hline Profissão do chefe da casa & & & & & & & \\
\hline Outros* & 05 & 17,9 & 03 & 60,0 & 02 & 40,0 & 0,75 \\
\hline Lavrador & 23 & 82,1 & 12 & 52,2 & 11 & 47,8 & \\
\hline Renda Familiar & & & & & & & 0.01 \\
\hline < 1 salário mínimo & 16 & 57,1 & 5 & 31,3 & 11 & 68,7 & \\
\hline$\geq 1$ salário mínimo & 12 & 42,9 & 10 & 83,4 & 02 & 16,6 & \\
\hline Renda per capita mensal & & & & & & & 0,60 \\
\hline$\leq 1 / 2$ salário mínimo & 24 & 85,7 & 11 & 45,8 & 13 & 54,2 &, 00 \\
\hline > 1/2 salário mínimo & 04 & 14,3 & 04 & 100 & 0 & 0 & \\
\hline
\end{tabular}

1 Teste Exato de Fisher. * Aposentado, conservador de estrada, lavrador e artesão. ** Não comparável pelo teste aplicado.

No relatório do Ministério da Saúde (PNDS, 2006) ${ }^{[18]}$, ao ser analisada a situação de SA e IA dos domicílios onde havia algum morador inscrito em programas sociais de transferência de renda (TR), foi observado que estes apresentavam prevalências superiores de IA, comparativamente aos demais. Domicílios urbanos com recebimento de TR apresentaram uma prevalência de IAM ou IAG maior que os rurais $(37,1 \%$ versus $31,3 \%)$. Assim, é possível que os diversos programas de TR estejam, de fato, incluindo entre seus usuários os grupos populacionais de muito baixo ingresso ou com outras características que conferem maior vulnerabilidade para a insegurança alimentar.
Em relação à profissão do chefe da família, observou-se que a maioria era formada por lavradores, este resultado já era esperado por ser Galheiros uma comunidade rural. Quanto à renda familiar, observou-se que a maioria das famílias $(57,1 \%)$ recebia menos que um salário mínimo (SM) mensal e que esta estava associada com a situação de insegurança alimentar $(p<0,01)$. Entre as famílias com renda familiar inferior a um SM, a prevalência de IA foi $68,7 \%$, já para as com ganho maior ou igual a um SM o valor encontrado para IA foi de $16,6 \%$. Tal fato pode ser o explicativo para o recebimento dos benefícios oriundos da União e do Estado (Tabela 3). 
$\mathrm{O} \mathrm{IBGE}^{[19]}$ considera a renda per capita mensal inferior a $1 / 2$ SM mensal como parâmetro para definição da linha de pobreza. Tomando este valor como referência, pode-se observar que $85,7 \%$ das famílias viviam nesta condição, resultado similar ao encontrado no estudo de Pereira et $a l^{[13]}$, no qual $95,1 \%$ das famílias declararam ter renda per capita mensal inferior a $1 \frac{1}{2}$ salário mínimo.Constatase, ainda, que a partir de $1 / 2$ SM per capita não há situação de IA, diferentemente do encontrado na PNAD/2004 ${ }^{[5]}$ (Tabela 3).

Não houve associação significativa entre a situação de segurança alimentar e a renda per capita $(p=0,60)$ entre as famílias estudadas, embora esta seja considerada a principal determinante de IA. Hoffmann ${ }^{[20,21]}$ observou que a residência rural, a atividade agrícola e a presença de pessoas com menos de 18 anos no domicílio foram contribuintes para redução da probabilidade de insegurança alimentar, variáveis estas presentes entre as famílias de Galheiros.
Em relação às condições de moradia e saneamento, nota-se pela Tabela 4 que a maioria das famílias residia em casa própria (96,4\%), de alvenaria incompleta $(92,9 \%)$ e que $67,9 \%$ utilizavam o poço e a fossa (arranjos particulares) para captação de água e destino dos dejetos, respectivamente, e que não houve associação entre estas variáveis e a situação de insegurança alimentar. Os valores encontrados para os serviços públicos de tratamento de água e esgotamento sanitário entre as famílias com IA $(52,6 \%)$ foram superiores aos encontrados na Paraíba $(47,0 \%)^{[15]}$.

Quanto aos bens materiais presentes nas casas de Galheiros, todas dispunham de geladeira em suas residências, $89,3 \%$ de fogão a gás e televisão e $63, \%$ de aparelho de som. Tais resultados indicam que esta comunidade detém o mínimo de SA no que se refere ao aspecto de conservação dos alimentos, por outro lado, apesar de ser rural, já incorporou o estilo de vida urbana.

Tabela 4. Distribuição das famílias de Galheiros, de acordo com as variáveis condições de moradia, de saneamento básico e bens materiais. Galheiros, Minas Gerais, 2006.

\begin{tabular}{|c|c|c|c|c|c|c|c|}
\hline \multirow[t]{2}{*}{ Variáveis estudadas } & \multicolumn{2}{|c|}{ Total } & \multicolumn{2}{|c|}{ AS } & \multicolumn{2}{|c|}{ IA } & \multirow[t]{2}{*}{ Valor $p$} \\
\hline & $\mathrm{n}$ & $\%$ & $\mathrm{n}$ & $\%$ & $\mathrm{n}$ & $\%$ & \\
\hline Tipo de construção da moradia & & & & & & & 0,21 \\
\hline Alvenaria completa & 02 & 7,1 & 0 & 0 & 02 & 100 & \\
\hline Alvenaria incompleta & 26 & 92,9 & 15 & 57,7 & 11 & 42,3 & \\
\hline Situação da moradia & & & & & & & 0,46 \\
\hline Própria & 01 & 3,6 & 0 & 0 & 01 & 100,0 & \\
\hline Cedida & 27 & 96,4 & 15 & 55,6 & 12 & 44,4 & \\
\hline Saneamento & & & & & & & 1,00 \\
\hline Outros* & 09 & 32,2 & 05 & 55,5 & 04 & 44,4 & \\
\hline Fossa e Poço & 19 & 67,9 & 10 & 52,6 & 09 & 47,4 & \\
\hline Bens & & & & & & & \\
\hline Geladeira & 28 & 100,0 & 15 & 53,6 & 13 & 46,4 & \\
\hline Fogão a gás & 25 & 89,3 & 14 & 56,0 & 11 & 44,0 & 0,58 \\
\hline Televisão & 25 & 89,3 & 15 & 60,0 & 10 & 40,0 & 0,21 \\
\hline Aparelho de som & 18 & 63,3 & 10 & 55,6 & 08 & 44,4 & 1,00 \\
\hline
\end{tabular}

1 = Teste Exato de Fisher, *fossa e água encanada e/ou esgoto e poço.

Embora a EBIA seja um instrumento

validado nacionalmente, ela apresenta certas limitações quanto aos diferentes aspectos da segurança alimentar, sendo assim, optamos por 
utilizar, concomitantemente à aplicação da mesma, outro indicador que demonstrasse os aspectos qualitativos do consumo alimentar complementando os resultados obtidos pela escala.

A distribuição das famílias, segundo a situação de segurança/insegurança alimentar e consumo diário de alimentos, encontra-se na Tabela 5. Nota-se que houve associação entre insegurança alimentar e consumo de alimentos apenas para o grupo das verduras $(p=0,03) \mathrm{e}$ para suco de frutas $(p=0,02)$. Esse resultado difere do encontrado por Marín-Leon et $a l^{[22]}$, os quais observaram diferenças significativas entre nível de insegurança alimentar e ingestão de alimentos dos grupos das carnes, frutas e leite. É interessante observar que para grupos alimentares mais caros, como por exemplo, a carne, não foi encontrada diferença estatística para situação de insegurança alimentar, isso demonstra a importância atribuída a esse alimento.

Pela Tabela 5 percebe-se ainda que apesar de não ter sido encontrada diferença estatística entre insegurança alimentar e consumo dos grupos das carnes, ovos, legumes e frutas; uma maior proporção de frequiência de consumo de cinco ou mais vezes por semana foi encontrada entre os com segurança alimentar. Esse resultado reforça a afirmação de Claro et $a l^{[23]}$, os quais relatam que a redução de preço de frutas, legumes e verduras, possível de ser obtida por meio de políticas públicas, poderia aumentar a participação desses alimentos na dieta dos brasileiros.

De acordo com o relatório do IBASE $^{[12]}$, as famílias beneficiadas com o
Programa Bolsa Família (PBF), realmente, passaram a adquirir mais alimentos, o que propiciou aumento do consumo, tanto em relação à quantidade, quanto à variedade dos mesmos. Entre as famílias que tinham a alimentação básica "suprida", o $\mathrm{PBF}$ possibilitou aumento na aquisição de alimentos considerados "complementares", como frutas, verduras, legumes, alimentos industrializados e outros considerados supérfluos, e também o consumo da carne, alimento valorizado e de difícil acesso. Entre as famílias que não tinham alimentação básica suprida, o PBF possibilitou que passassem a comprar mais alimentos considerados básicos, como feijão e arroz. Acredita-se que tal fato ocorreu em Galheiros, uma vez que todas as famílias relataram ingerir, diariamente, arroz, feijão e óleos.

Para os autores do relatório citado, anteriormente, é necessário manter e aprofundar o PBF, associando-o a outras políticas públicas capazes de atacar problemas como a falta de saneamento básico e de acesso ao mercado formal de trabalho - fatores que guardam correlação com a insegurança alimentar. Oferta de alimentos mais baratos, ampliação da alimentação escolar para o ensino médio, entre outras, são também políticas governamentais que poderiam contribuir para uma melhora desses índices.

A ausência de associação estatisticamente significativa entre as variáveis, renda per capita, recebimento de benefício governamental, freqüência de consumo alimentar, presença de moradores menor de 18 anos, e a situação de segurança alimentar, deve-se, provavelmente, ao pequeno tamanho da amostra. Outro fator relevante é 
que trata - se de uma comunidade rural, e apesar do elevado número de famílias com baixa renda, a lavoura, os programas de benefício do Governo, a existência de um comércio de produtos alimentícios, no qual há sempre débitos a serem quitados, podem estar contribuindo para os resultados encontrados.

Tabela 5. Distribuição das famílias segundo a freqüiência de consumo semanal de alguns grupos de alimentos e situação de segurança alimentar da comunidade de Galheiros, Minas Gerais, 2006.

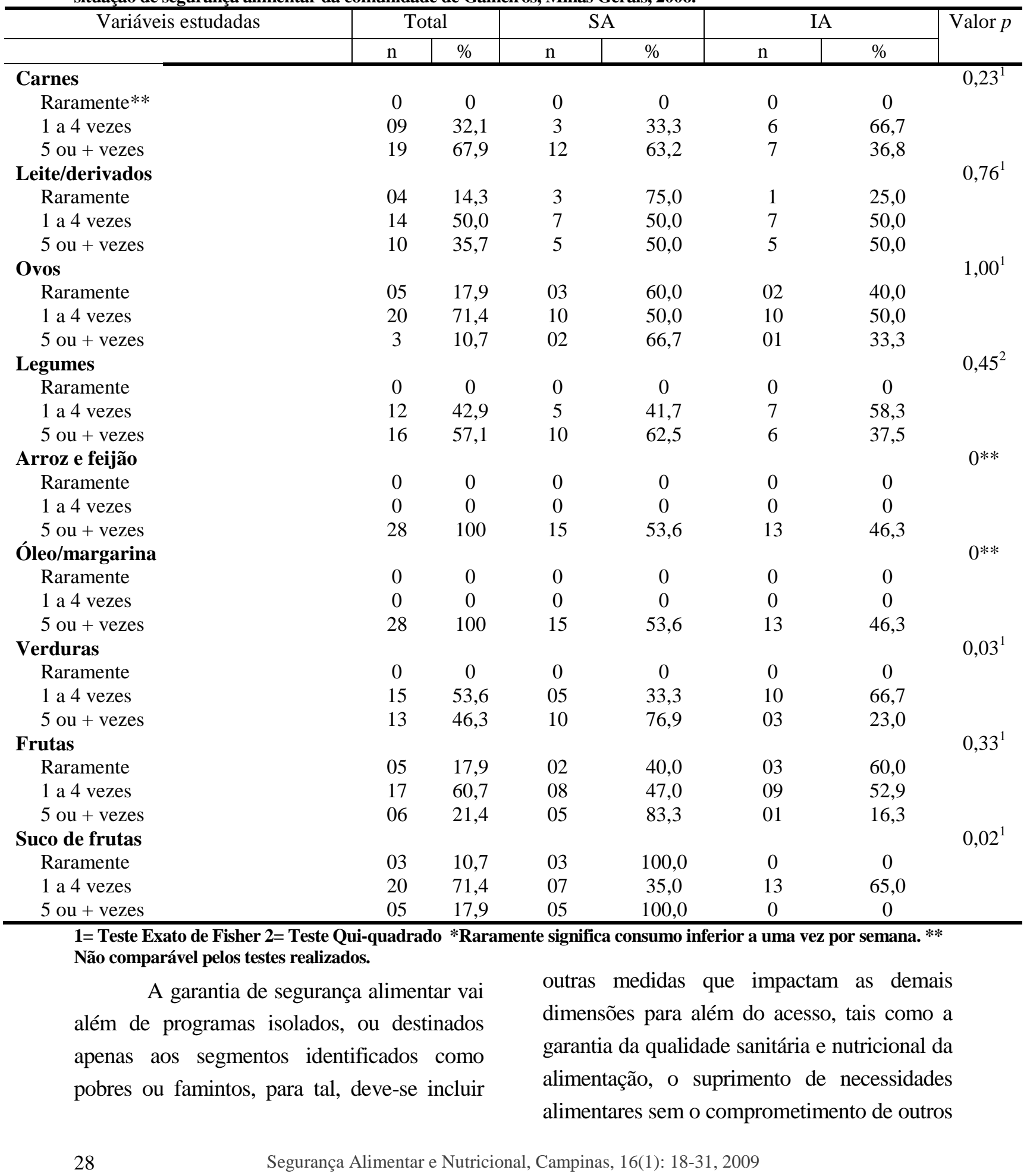


gastos, igualmente essenciais, e um modelo de produção de alimentos sustentável do ponto de vista ambiental e socioeconômico ${ }^{[24]}$.

\section{Conclusão}

Constata-se que o acesso a alimentos em quantidade e qualidade adequados ao consumo, ainda é um obstáculo a ser ultrapassado pela comunidade estudada, uma vez que, parte da comunidade convive com a insegurança alimentar, ou seja, com o medo de ficar sem alimentos, ou mesmo, com a situação de fome. Tal situação é vivenciada por metade das famílias que tinham moradores com idade inferior a 18 anos, sendo que a maioria vivia com uma renda familiar inferior a um salário mínimo, e metade recebia algum benefício do Governo Estadual/Federal. Esses resultados demonstram que apesar de existirem no Brasil políticas públicas de combate à fome, grande parte das famílias residentes em Galheiros, vive em vulnerabilidade social. Assim sendo, para reverter tal quadro, serão necessárias ações estruturantes voltadas à produção, acesso e consumo de alimentos em qualidade e quantidade suficientes.

\section{Agradecimento}

Os autores agradecem o Conselho Nacional de Desenvolvimento Científico e Tecnológico pelo financiamento da pesquisa (CNPq - Processo n. 553332/2005-8).

\section{Referências Bibliográficas}

[1] Conselho Nacional de Segurança Alimentar e Nutricional - CONSEA. Princípios e diretrizes para uma política nacional de segurança alimentar. Brasília: CONSEA, 2004.BRASIL.
[2] Valente FLS. O combate à Fome e a desnutrição e a promoção da alimentação adequada no contexto do Direito Humano à Alimentação: um eixo estratégico do desenvolvimento humano sustentável, 2003. 38p. Mimeo.

[3] Conselho Nacional de Segurança Alimentar e Nutricional - CONSEA. A construção de uma política de Segurança Alimentar e Nutricional. II Conferência Nacional de Segurança Alimentar e Nutricional. Textos Base. Brasília: CONSEA, 2004.

[4] Maluf RS, Zimmermann S. Políticas municipais de erradicação da fome e a promoção da agricultura familiar no Brasil. R. Janeiro， CERESAN/UFRRJ， 2005， 19 p. (Relatórios Técnicos, 1).

[5] Fundação Instituto Brasileiro de Geografia e Estatística (IBGE). Pesquisa Nacional por Amostra de Domicílios 2004: Segurança Alimentar. Rio de Janeiro; 2006.

[6] Bickel G, Nord M, Price C, Hamilton W, Cook J. Measuring Food Security in the United States: Guide to measuring household food security. USDA, Office of Analysis, Nutrition and evaluation, USA, 2000. 82p

[7] Segall-Corrêa AM, Perez-Escamilla R, Sampaio MF, Marin L, Panigassi G, Maranha LK, Acompanhamento e avaliação da Segurança Alimentar de famílias brasileiras: validação de metodologia e de instrumento de coleta de informação. Relatório Técnico, Campinas SP; Março de 2004.

[8] Sampaio MFA, Kepple AW, Segall-Corrêa AM, Oliveira JT, Panigassi G, Maranha LK, 
Marin-Leon L, Bergamasco SMPP, PerezEscamilla R. (In) Segurança Alimentar: experiência de grupos focais com populações rurais do Estado de São Paulo. Segurança Alimentar e Nutricional. 2006; 13 (1):64-77.

[9] Yuyama LKO, Aguiar JPL, Pantoja L, Maeda RN, Melo T, Alencar FH, Nascimento AMM, Negreiros NMA, Segall-Corrêa AM, Pérez-Escamilla R. Segurança/insegurança alimentar em famílias urbanas e rurais no estado do Amazonas: I. Validação de metodologia e de instrumento de coleta de informação. Acta Amazonica, 2007;37(2): 247-252.

[10] Segall-Corrêa AMS. Insegurança alimentar medida a partir da percepção das pessoas. Estudos Avançado. 2007; 21 (60):143-154.

[11] Pereira DA, Vieira VL, Fiore EG, Cervato-Mancuso AM. Insegurança Alimentar em Região de Alta Vulnerabilidade Social da Cidade de São Paulo. Segurança Alimentar e Nutricional, 2006; 13(2): 34-42.

[12] Instituto Brasileiro de Análises Sociais e Econômicas (IBASE). Documento síntese: Repercussões do Programa Bolsa Família na Segurança Alimentar e Nutricional das Famílias Beneficiadas, 2008, p1-20.

[13] Galeazzi MAM, Bonvino H, Lourenço, F, Vianna, RPT. Inquérito de Consumo Familiar de Alimentos. - Metodologia para Identificação de Famílias de Risco Alimentar. Cadernos de Debate, 1996; vol. IV: 32-46.

[14] SAS Institute (1990) SAS/STAT® user's guide: version 6. 4ed. Cary, NC.
[15] Vianna RPT, Segall-Corrêa. Insegurança alimentar das famílias residentes em municípios do interior do estado da Paraíba, Brasil. Revista de Nutrição.2008; 21 (Suplemento):111-122.

[16] Sen A. Poverty and Famines. An essay on entitlement and Deprivation. Oxford: Clarendon Press; 1981.

[17] Hoffmann R. Determinantes da insegurança alimentar no Brasil. In: Anais do 14o Congresso da Sociedade Latinoamericana de Nutrición; 2006; Florianópolis, BR.

[18] Ministério da Saúde. Pesquisa Nacional de Demografia e Saúde da Criança e da Mulher PNDS 2006: Relatório. Brasília/DF 2008. p.263-275.

[19] IBGE Instituto Brasileiro de Geografia e Estatística. Condições de Pobreza. [documento na

Internet]. Disponível em: http:/www.ibge.gov.br/ibgeteen/glossário/pobr eza.html. Acesso em 30 maio 2008.

[20] Hoffmann R. Pobreza, insegurança alimentar e desnutrição no Brasil. Estudos Avançados. 1995; 9(24): 159-72.

[21] Hoffmann R. Determinantes da Insegurança Alimentar no Brasil: Análise dos Dados da PNAD de 2004. Segurança Alimentar e Nutricional, 2008;15(1):49-61.

[22] Marín-León L, Segall-Corrêa AM, Panigassi G, Maranha LK, Sampaio MF, Perez-Escamilla R. A percepção de insegurança alimentar em famílias com idosos em Campinas, São Paulo, Brasil. Cadernos de Saúde Pública. 2005; 21(5):1433-1440. 
[23] Claro RM, Carmo HCE, Machado FMS, Monteiro CA. Renda, preço dos alimentos e participação de frutas e hortaliças na dieta. Revista de Saúde Pública 2007;41(4):557-64.

[24] Burlandy L. Transferência condicionada de renda e segurança alimentar e nutricional. Ciência \& Saúde Coletiva, 2007;12(6):14411451. 